ARTICLE

\title{
Hadrontherapy: a Geant4-Based Tool for Proton/Ion-Therapy Studies
}

\author{
G. A. Pablo CIRRONE ${ }^{1,}$, Giacomo CUTTONE ${ }^{1}$, S. Enrico MAZZAGLIA ${ }^{1}$, Francesco ROMANO ${ }^{1}$, \\ Daniele SARDINA ${ }^{1}$, Clementina AGODI $^{1}$, Andrea ATTILI ${ }^{2}$, A. Alessandra BLANCATO ${ }^{1}$, \\ Marzio DE NAPOLI ${ }^{1}$, Francesco DI ROSA ${ }^{1}$, Pekka KAITANIEMI ${ }^{3}$, Flavio MARCHETTO ${ }^{2}$, \\ Ivan PETROVIC ${ }^{4}$, Aleksandra RISTIC-FIRA ${ }^{4}$, Jungwook SHIN ${ }^{5}$, \\ Nikolai TARNAVSKY ${ }^{6}$, Stefania TROPEA ${ }^{1}$ and Christina ZACHARATOU ${ }^{6}$ \\ ${ }^{1}$ Laboratori Nazionali del Sud of INFN (Italian Institute for Nuclear Physics), 95100 Catania, Italy \\ ${ }^{2}$ Turin Section of the INFN (Italian Institute for Nuclear Physics), 10121 Turin, Italy \\ ${ }^{3}$ Helsinky Institute of Physics, 00-02, Helsinky, Finland \\ ${ }^{4}$ Vinca Institute of Nuclear Sciences, University of Belgrade, 11000 Belgrade, Serbia \\ ${ }^{5}$ National Cancer Center, Seul, Korea \\ ${ }^{6}$ Niels Bohr Institute, 1000 Copenhagen, Denmark
}

\begin{abstract}
Hadrontherapy is a $\mathrm{C}++$, free and open source application developed using the Geant4 Monte Carlo libraries. The basic version of Hadrontherapy is contained in the official Geant4 distribution (www.cern.ch/Geant4/download), inside the category of the advanced examples. This version permits the simulation of a typical proton/ion transport beam line and the calculation of dose and fluence distributions inside a test phantom.

A more complete version of the program is separately maintained and released by the authors and it offers a wider set of tools useful for Users interested in proton/ion-therapy studies. It gives the possibility to retrieve ion stopping powers in arbitrary geometrical configuration, to calculate 3D distributions of fluences, dose deposited and LET of primary and of the generated secondary beams, to simulate typical nuclear physics experiments, to interactively switch between different implemented geometries, etc.

In this work the main characteristics of the actual full version of Hadrontherapy will be reported and results discussed and compared with the available experimental data.

For more information the reader can refer to the Hadrontherapy website.
\end{abstract}

KEYWORDS: Hadrontherapy, Monte Carlo simulation, Geant4

\section{Introduction}

At the Laboratori Nazionali del Sud of the Instituto Nazionale di Fisica Nucleare (INFN-LNS), in Catania (I), the first Italian hadrontherapy facility named CATANA (Centro di AdroTerapia ed Applicazioni Nucleari Avanzate) has been realized. ${ }^{1)}$

The facility makes use of $62 \mathrm{MeV}$ proton beams accelerated by a supercounducting cyclotron and transported inside a specific treatment room to be used for the radiotherapeutic treatment of some kinds of ocular tumours. By the end of 2009 , about 77,300 patients worldwide have been treated with proton and 7,100 with carbon radiotherapy. Of these, about 16,800 have been treated for ocular diseases.

The CATANA facility is based on a passive transport beam line where the original accelerated proton beams are shaped (in spatial and energy distribution) by physical devices placed along the line.

Inside the treatment room, the beam exits in air throughout a thin kapton window and traverses a set of different transport and diagnostic elements until it finally reaches the

*Corresponding author, E-mail: cirrone@1ns.infn.it

(C) 2011 Atomic Energy Society of Japan, All Rights Reserved. patient, located three meters downstream the kapton. Since March 2002, 212 patients have been treated inside the CATANA facility. A more detailed description of the CATANA therapeutic beam line can be found elsewhere. ${ }^{1)}$

The described proton treatment beam line is not exclusively used for the patients' treatment but it also represents an optimal irradiation point for detectors' test, radiation damage studies and radiobiological experiments.

In addition to the proton irradiation point, a second experimental room at INFN-LNS is dedicated to in-air multidisciplinary irradiation studies. Also this second beam line is used by many researchers groups in different kinds of experiments (detector tests, irradiation of biological samples, etc.). In this second beam line different ion beams can be transported with an energy ranging from $70 \mathrm{AMeV}$ down to about $20 \mathrm{AMeV}$.

Even if the first patient has been treated with hadrons more than 40 years ago, the use of proton and ion beams for clinical applications can be still considered as pioneer activity and it is evident since this technique can still reach further improvements in the future. The intense research activity in this field is demonstrated by a huge number of scientific publications constantly produced and related to the 
improvement of the transport beam lines, of the dosimetry, of the treatment planning algorithms as well as to a better understanding of the radiobiological effects of ions.

Our research group, on the basis of the experience so far gained in the realisation of the CATANA facility and considering the future hadron-therapy centres that will be developed in Europe and around the world in the next years, decided to start an R\&D program in the framework of the INFN (Instituto Nazionale di Fisica Nucleare)-Geant4 collaboration.

The main goal of this activity, originally started in 2003, is the development of a free and open source Monte Carlo application. It is named Hadrontherapy and is entirely constructed using the libraries of the Geant4 simulation toolkit. ${ }^{2,3)}$

The Monte Carlo method can play a very important role in many aspects of the clinical use of protons and ions. The dose prediction algorithms used today for the ocular proton therapy treatment planning, for example, rely on parameterizations of measured proton dose distribution (broad beam approach, pencil beam approach, etc.), whose predictive capabilities are limited by the approximations and simplifications adopted in these models. On the opposite, the Monte Carlo technique can provide, in principle, a very accurate prediction of the proton treatment beams by taking into account all the physics processes involved, including electromagnetic energy loss, energy straggling, multiple Coulomb scattering, elastic and non elastic nuclear interactions as well as the transport of any generated secondary particle. Of course, to take full advantage of the Monte Carlo approach, the beam delivering system has to be simulated in detail and the beam initial parameters have to be known as accurate as possible.

In this context we developed Hadrontherapy, ${ }^{4}$ firstly publicly released in the 2004 inside the Geant 4 distribution and today furnished in two different versions: the basic one, still contained inside Geant4 in the Advanced Examples category, and the full one, more complete and separately distributed by the authors at a specific web site. ${ }^{5)}$ In the same web pages the installation instructions and the documentation can be found.

In this paper we will show and discuss the main characteristics and potentialities of the full version of Hadrontherapy.

\section{History and General Description of the Hadron- therapy Application}

Hadrontherapy is a free and open source application, regularly maintained and improved by some of the authors of this paper. It permits, in its simplest version, the simulation of a typical beam line for proton/ion therapy including all the necessary transport elements: the diffusion and modulation systems for the particles spatial and energy distribution, the collimators, the transmission detectors as well as detectors for the dose distribution measurements, which can be simulated and activated via simple and external commands.

Since the beginning of its development Hadrontherapy has undergone many changes and its complexity and capa- bilities have grown to such an extent that in 2009 the authors, decided to release a specific version separated from the one inserted as Geant4 example. This decision appeared necessary in order to still maintain a basic version of the program but at the same time, to offer to the hadrontherapy users a complete tool to face the complex problematic of the proton/ion therapy world.

This full version offers many additions with respect the basic one. Among them the most important are the modularization of the geometry, that gives the possibility to change the simulated apparatus interactively and externally, via script commands; the modularization of the applicable physics models permitting the User to choose among the best physics models available in Geant 4 for proton/ion applications; the possibility to easily calculate stopping powers and ranges (this latter function is not yet present in the last stable version) in simple geometrical configurations and for any couple of ion-material combinations; the presence of specific algorithms for the calculation of the average 3-dimensional LET (Linear Energy Transfer); a new graphical user interface, implemented using the QT libraries, that can be chosen (if installed) in order to have a more simple interaction with the program. Moreover, the next versions of the application will include the possibility to use DICOM interfaces in order to take into account the different tissue densities.

\section{Geometry Description}

The geometrical module Hadrontherapy is divided in two main independent blocks, both realized with one or more specific class files: the block delegated to the simulation of the geometry and that one reserved to the simulation of a detection region, i.e. a specific volume, with dimensions, position and material that can be easily changed, permitting the scoring of the specific quantities of interest (dose deposited, particle fluences, LET distribution, etc.). This region, generally called "phantom" in the medical physics slang, can be sliced or voxelized (with arbitrary dimensions of slices and voxels) to permit a 2D- or 3D-scoring

An important capability, recently added in Hadrontherapy, is the extreme simplicity to change between different transport beam lines. The User can, in fact, via external macro commands, decide to use different geometrical set-ups while maintaining unchanged detector part. In the latest public version of the program we provide two beam lines in operation at INFN-LNS and previously described and a geometry set-up specifically designed to perform nuclear physics experiments and calculate the most important nuclear physics related quantities (like total or differential production cross sections). This geometry is used from some of the authors of this paper to perform specific validation of the implemented nuclear models, in order to test the actual capability of Geant 4 in the framework of the ion therapy applications.

In the near future a dedicated module for the simulation of an active-scanning system for proton-therapy will be added.

\section{Physics Description}

Hadrontherapy is characterised by a very simple interface to implement more appropriate physics models and it makes 
use of the so-called Physics Lists and Reference Physics Lists. These are set of pre-compiled and ready-to-use set of physics models contained in the installation directory of the Geant4 code (inside the folder <installation folder $>$ /source/physicslist/).

The Physics List contains a set of physics models. There are Physics Lists for the electromagnetic physics, for hadronic elastic and for hadronic inelastic physics, etc. The Reference Physics List can be considered as a collection of Physics Lists and can contain both the electromagnetic as well as the hadronic models.

In Hadrontherapy we suggest the use of the QGSP_BIC_EMY Reference Physics List that has been required and tested by some of the authors of the present paper. It has been specifically created to address simulation problems for which high level of accuracy is requested. QGSP BIC EMY is an acronym that briefly explain all the physics models activated when it is called: QGSP (Quark Gluon String Precompound) defines the hadronic models for nucleons; BIC (Binary Ion Cascade) defines the inelastic models for ions and EMY (ElectroMagnetic Y) defines the electromagnetic models used by all the particles ( $\mathrm{Y}$ indicates a particular EM physics particularly tailored for the use in medical physics.

All the results presented in this paper and that are relative to the simulation of the proton beams have been obtained using the QGSP_BIC_EMY. As the Reference Physics List capable to correctly handle the inelastic interactions of ion beams is not yet available in Geant4, we preferred to adopt the Physics Lists approach for the carbon beams simulations. In this case, in fact, we activated all the Physics Lists already contained in the QGSP_BIC_EMY but adding the G4BinaryLigthIon Physics List, able to handle the ion-ion inelastic interaction.

\section{Simulations Configuration}

All the results presented in this work have been obtained with the 9.3 (patch 1) version of Geant 4 and simulate in details the two INFN-LNS irradiation beam lines briefly described in the introduction. The scoring of dose, fluence and LET have been performed using a cubic voxelized phantom placed at the end of the beam line, exactly in the point where the patient is located in the real treatment. The phantom has been divided in cubic voxels each with dimension of $10 \times 10 \times 10$ micrometers.

Figure 1 shows the Geant4 simulation output of the carbon irradiation beam line. The blue tracks represent the carbon ions traversing the beam line and reaching the phantom (red cube). Inside the phantom a smaller cubic volume (in cyan) is the sensible voxelized detector.

The initial characteristics of the beams have been defined matching the characteristics (peak to plateau ratio, Full Width Half Maximum, peak position, in particular) of the simulated Bragg peak distribution with the experimental data In both proton and carbon case the primary beam is generated from a circular spot from which particles are emitted with a gaussian spatial distribution (standard deviation of $1 \mathrm{~mm}$ ). The initial energy distribution of the beams is gaussian, with

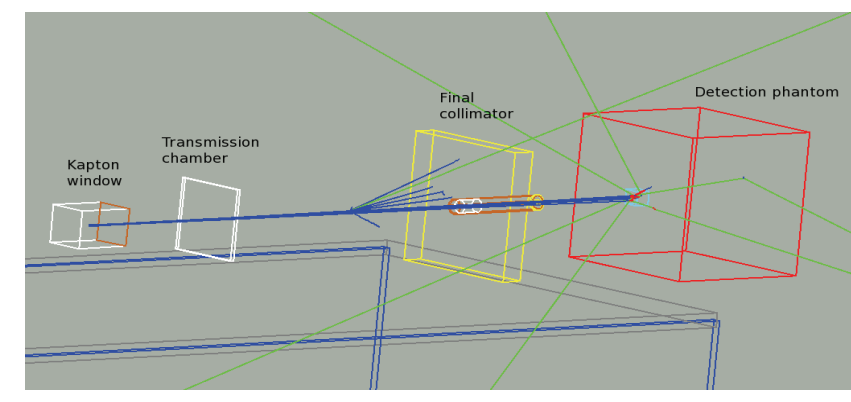

Fig. 1 Graphic simulation output of the LNS-INFN carbon transport beam line

a standard deviation (defining the energy spread of the beam) of $0.3 \%$ in both proton and carbon cases. No divergence of the primary beam has been considered, in agreement with the cyclotron specification.

\section{Calculations and Comparisons with Measure- ments}

\section{Proton/Carbon Dose Distributions}

Comparisons between experimental and simulated depth dose distributions (Bragg peak) are necessary in order to verify and validate the developed code. For the specific case of Bragg peak comparisons, the cubic phantom has been divided in 4,000 slices, 10 um of thickness, orthogonal to the beam direction, inside which the total dose is retrieved. In order to have a reasonable statistical fluctuation (less than $3 \%$ ) without huge calculation times, the simulation transport parameters must be carefully chosen. We decided to use a production cut of $0.01 \mathrm{~mm}$ and a Max-Step of $0.01 \mathrm{~mm}$. The production cut determines how many secondary electrons and gamma are transported by the application (lower is its value bigger is the number of secondary and the resulting total computation time); on the other hand, the Max-Step parameter forces the step performed by any particle during its propagation: also in this case a small step means a big precision in the transportation but a long computation time. The data obtained by the simulation have been compared with data acquired at INFN-LNS in Catania for both proton and carbon ion beams.

Two experiments have been performed for this purpose: protons and carbon ions accelerated at $62 \mathrm{AMeV}$ are respectively transported in the two experimental rooms, already described before. In the two cases the same acquisition system has been used for the measurement of the released dose. It mainly consists of a parallel plate ionization chamber, having an active volume of $0.055 \mathrm{~cm}^{3}$, polarized with an electric field of $300 \mathrm{~V}$ and coupled with a dedicated electrometer. The chamber, placed in a cubic water phantom, is able to measure the depth dose distribution with a spatial resolution of $50 \mu \mathrm{m}$ by means of a motorized system.

Figure 2 reports the simulation of a typical clinical proton depth dose distribution obtained with Hadrontherapy and the corresponding experimental data. In Fig. 3 the comparison between the experimental and the Geant4 Bragg peak is showed for the carbon case. A satisfactory agreement has been found between the experimental and calculated curves 


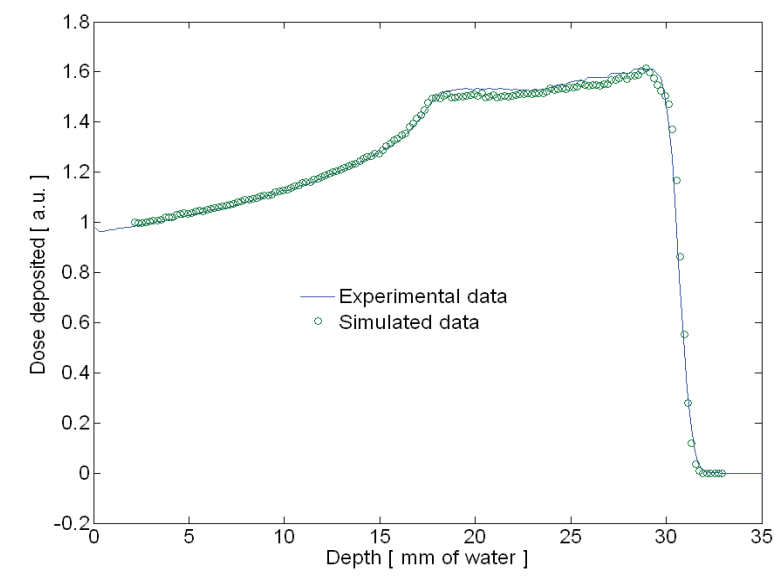

Fig. 2 Proton clinical depth dose distribution simulated with Hadrontherapy (dots) and experimentally measured (line). Experimental curve is acquired using a plane parallel ionisation chamber (Markus Chamber type) in water.

for both proton and carbon beams. These results demonstrate the accuracy of Hadrontherapy and Geant4 in the dose reconstruction.

\section{LET Calculations}

The Linear Energy Transfer (LET) is a measure of the energy transferred by an ionising particle traversing a material. Even if its definition is closely related to that of the stopping power (that measures the energy loss of an ionising particle per unit distance) the LET focuses only upon the energy transferred to the material surrounding the particle track by the secondary electrons.

The LET is typically used to quantify the effects of the ionising radiation on biological specimens or electronic device and it is usually expressed (like the stopping power) in $\mathrm{keV} / \mu \mathrm{m}$.

As one is usually interested in energy transferred to the material in the vicinity of the particle track, with this definition, the secondary electrons with energies larger than $\Delta$ are excluded: ${ }^{6}$ the electrons of high energy have a large range and this energy limit effectively does not consider electrons that travel far from the primary particle.

Following this, the LET (also called restricted linear electronic stopping power) is defined by:

$$
L_{\Delta}=\frac{d E_{\Delta}}{d x},
$$

where the $d E_{\Delta}$ is the energy loss due to electronic collisions minus the kinetic energies of all secondary electrons with an energy larger than $\Delta$.

This definition is exhaustive in case of monoenergetic beams. When the irradiation beam has different energy components (as in the case of a real clinical beam) the introduction of the concept of average LET is needed and the concept of LET distribution becomes more meaningful. Hence, mean LET values have to be considered which may be simply referred with the generic term 'LET'.

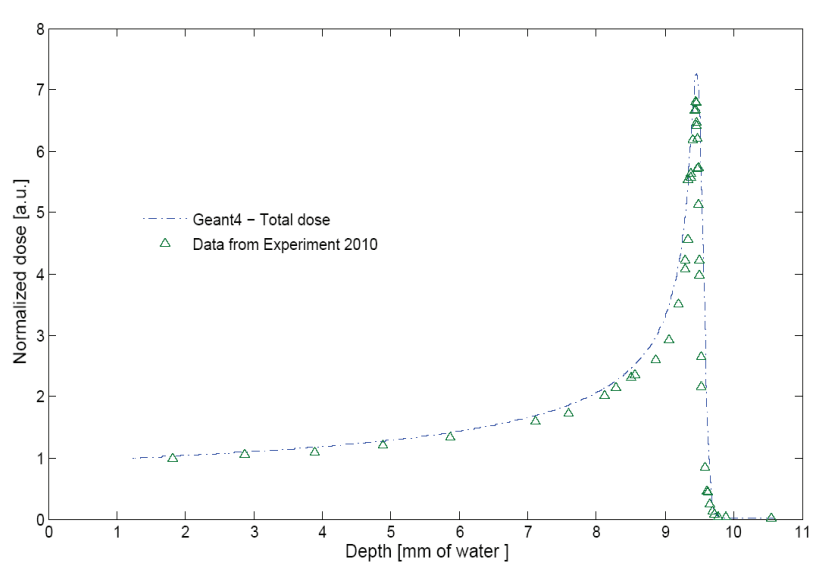

Fig. 3 Simulated and experimental depth dose profiles of a $62 \mathrm{AMeV}$ Carbon beam in water

The so defined LET is obtained averaging, at a given depth $z$, the particles stopping powers $S(E)$ where $E$ is the particle energy spectra at a given depth. There are two main common implementations of mean LET: the track averaged LET or LET track ${ }^{7,8)}$ and the dose averaged LET or simply LET dose. ${ }^{9)}$ The first one is the mean value $S(E)$ weighted on the particle fluence, the second one is instead weighted by its contribution on the local dose.

A module for the LET calculation has been recently implemented inside Hadrontherapy as a first step towards the introduction of a model for the radiobiological damage.

We developed an algorithm able to calculate the LET values versus the depth of a particle (and of the secondaries produced by its interaction) in the traversed material.

For proton incident beams, secondary particle production is not really significant in terms of fluence contribution. Hence, in this case, only the primary proton averaged track LET and dose LET have been computed. For ${ }^{12} \mathrm{C}$ ion beams the situation gets more complicated. In this case, indeed, inelastic interactions are no more negligible: a mixed radiation filed is created and different contributions in averaged LET distribution due to the produced isotopes have to be combined. Considering a specific isotope $\mathrm{j}$, the approach we have used consists on registering and scoring the kinetic energies of the particles $\mathrm{j}$ when they traverse a specific slab, located at the depth positions $\mathrm{z}$ inside the PMMA phantom. Because of the interactions suffered along the path, the beam traversing the medium is not mono-energetic but a local spectrum is present for each depth $\mathrm{z}$. The primary particles, as well as the secondary ones, are characterised by an energy spread, which increases with depth. In the Hadrontherapy application we have simulated this complex configuration and calculated the averaged track and dose LET. For each $\mathrm{j}$ particle the local spectrum of the kinetic energy is stored and saved in histograms using the ROOT ${ }^{10)}$ analysis program. Once the local energy spectra have been collected for each particle and depth, these information have to be linked with the corresponding stopping power values. In such a way, at the end of a simulation run, a depth distribution of LET track 


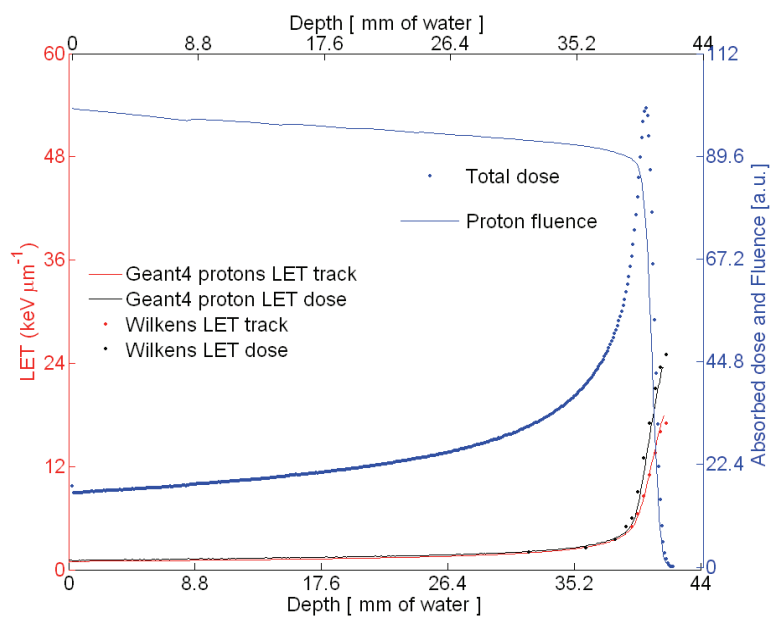

Fig. 4 Dose and track LET curves obtained with Hadrontherapy and compared with the data obtained by Wilkens for a $70 \mathrm{MeV}$ proton beam. In the right $\mathrm{y}$-axis, the corresponding proton dose and fluence values are represented.

and LET dose is obtained both for each single isotope $\mathrm{j}$ and for the total contribution due to all the isotopes present in the mixed radiation field.

In order to verify the correct implementation of the developed LET calculation methods, we firstly performed a comparison with a paper by Wilkens ${ }^{11)}$ reporting the track and dose LET values calculated with an analytic method. In Fig. 4 the curves represent the dose and track LET distributions obtained with Hadrontherapy (lines) and the ones obtained by Wilkens (dots), together with the total depth dose distribution and the protons fluence. Our results are in good agreement with Wilkens's ones. In both cases, as expected the average LET is practically constant and rather low in the entrance channel and, it rapidly increases in the proximity of the Bragg peak, reaching values bigger than $20 \mathrm{keV} / \mathrm{um}$. Moreover, dose LET is always higher than track LET.

Then, we performed a set of calculations of LET using the same described approach, but for the $62 \mathrm{AMeV}$ carbon beam case. As already mentioned, in case of carbon ion incident beams, secondary charged particles are produced and inelastic nuclear interactions have to be considered for the average LET calculations. In this case, at a specific depth $z$, the local energy spectra related to each produced isotope have been stored. As expected, the main contribution in both dose and track LET is mainly due to the primary carbon ions, even if the contributions of the secondary particles, which become more evident beyond the distal part of the peak, is not negligible. In Fig. 5 track and dose LET are shown for primary carbon ions, together with the respective fluence and the total depth dose distributions. In this case, calculations have been done in PMMA (polimethilmethachrilate) in order to simulate the exact configuration of a radiobiological experiments performed at LNS for the evaluation of human melanoma cell survival. The trend of the mean carbon LET is similar to the proton case but the absolute values are more than one order of magnitude higher.

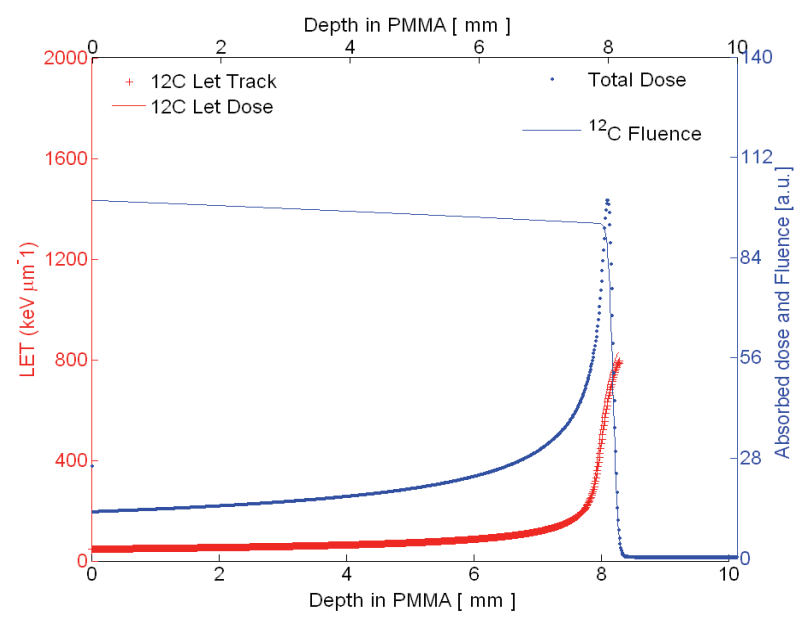

Fig. 5 Dose and track LET curves obtained with Hadrontherapy for the $62 \mathrm{AMeV}$ carbon beam. Only the primary beam is considered in the LET calculations. The normalised carbon depth dose and fluence curves are showed in blue and the right $\mathrm{y}$-axis expresses their values.

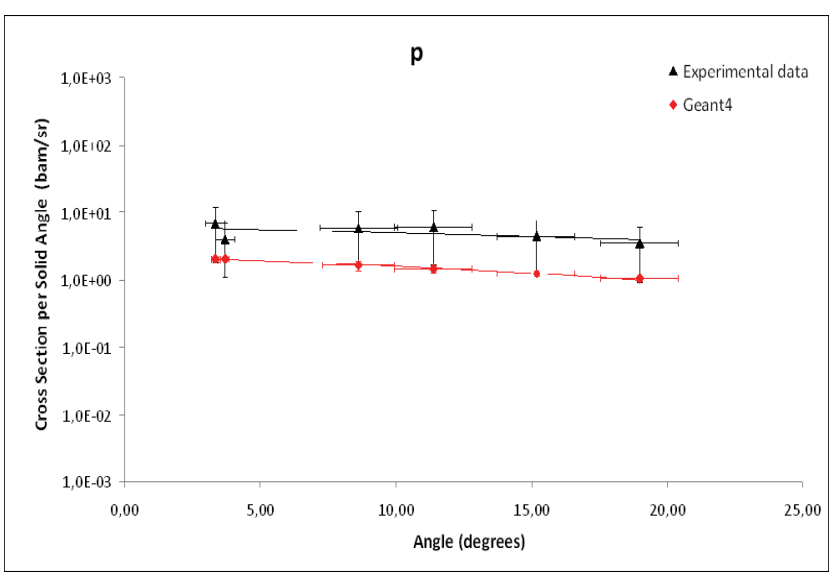

Fig. 6 Angular distributions of proton production cross section for ${ }^{12} \mathrm{C}$ ions at $62 \mathrm{AMeV}$ on gold target. Red line represents the Geant4 result while the black one is the experimental data. For the simulated data only the errors on angle definitions are considered.

\section{Fragments Production Cross Sections}

Especially in the case of radiation therapy with ions $(Z>1)$, the ability to predict the spectra of the produced secondary particles is of fundamental importance from the dosimetric as well as from the radiobiological point of view. Of course, the capacity of Monte Carlo method in this case, strictly depends on the reliability of the nuclear models implemented in the code. For this purpose an extensive model validation plan has been carrying out by our group in these years, which includes the verification of charged production cross sections for different carbon ion incident energies and target materials. Some measurements have been already carried out at LNS-INFN of Catania, in order to cover the gap of data at the energy range of interest in hadrontherapy (60$400 \mathrm{AMeV}$ ). Carbon ions are accelerated at $62 \mathrm{AMeV}$ and their interaction with thin ${ }^{197} \mathrm{Au},{ }^{12} \mathrm{C}$ and $\mathrm{CH}_{2}$ targets is studied. Produced charged nuclear fragments have been detected 
using two Si-CsI hodoscopes with different granularity, i.e. Hodo-small and Hodo-big, respectively composed by twofold and three-fold telescope detectors covering a total angle of $21.5^{\circ}$.

The experimental apparatus has been simulated with Geant4 as well as the initial parameters of the incident beam. Double differential cross sections and angular distributions have been calculated for each produced isotope and compared with the experimental data. Figure 6 shows an example of angular distributions comparison (protons, in the specific case) obtained in the interaction of ${ }^{12} \mathrm{C}$ with gold targets. The comparisons with data show that the trend of the distributions is generally well reproduced, even if differences in the absolute value are still present, especially for heavier fragments. Moreover, most of charged fragments are forward produced, with a large production of alpha particles, as expected.

More recent experiments have been also carried out for further investigations and analyses are still in progress. An experiment with a bigger and more complex experimental apparatus is also scheduled for 2011 at GSI (Darmstadt, Germany), for fragmentation measurements at higher energies (up to $1 \mathrm{AGeV}$ ) on different targets of medical interest.

\section{References}

1) G. A. P.Cirrone et al.," $62 \mathrm{AMeV}$ proton beam for the treatment of ocular melanoma at Laboratori Nazionali del Sud -
INFN,” IEEE Trans. Nucl. Sci., 51[3], 860-865 (2004).

2) S. Agostinelli et al., "Geant4 - a simulation toolkit", Nucl. Instr. Meth. Phys. Res., A536[3], 250-303 (2003).

3) J. Allison et al., "Geant4 developments and applications," IEEE Trans. Nucl. Sci., 53[1],, Part 2, 270 (2006).

4) G. A. P. Cirrone et al., "Implementation of a new Monte Carlo - GEANT4 simulation tool for the development of a proton therapy beam line and verification of the related dose distributions," IEEE Trans. Nucl. Sci., 52, 262-265 (2005).

5) https://sites.google.com/site/hadrontherapy/

6) Fundamental Quantities and Units for Ionizing Radiation, Technical Report 60, International Commission on Radiation Units and measurements (ICRU), Bethesda, MD, (1998).

7) Linear Energy Transfer, Technical Report 16, International Commission on Radiation Units and measurements (ICRU), Washington DC, (1970).

8) E. J. Hall et al., "The relative biological effectiveness of 160 MeV protons," Int. J. Radiat. Oncol. Biol. Phys., 4, 1009-13 (1978).

9) M. J. Berger, Penetration of Proton Beams Through Water I. Depth-dose Distribution, Spectra and LET Distribution, NISTIR Publication 5226 National Institute of Standards and Technology, Gaithersburg, MD, (1993).

10) R. Brun, F. Rademakers, "ROOT - An object oriented data analysis framework", Nucl. Instr. Meth. Phys. Res., A389[1-2], 81-86 (1997).

11) J. J. Wilkens, U. Oelfke, "Analytical linear energy transfer calculations for proton therapy," Med. Phys., 30[5], 806-815 (2003). 